\title{
Synthesis and antioxidant activity of long chain alkyl hydroxycinnamates
}

\author{
Jose C.J.M.D.S. Menezes ${ }^{\mathrm{a}}$, Shrivallabh P. Kamat ${ }^{\mathrm{a}, *}$, Jose A.S. Cavaleiro ${ }^{\mathrm{b}}$, Alexandra Gaspar ${ }^{\mathrm{c}}$, \\ Jorge Garrido ${ }^{\mathrm{c}, \mathrm{d}}$, Fernanda Borges ${ }^{\mathrm{c}, *}$ \\ ${ }^{a}$ Department of Chemistry, Goa University, Taleigao Goa 403 206, India \\ ${ }^{\mathrm{b}}$ Department of Chemistry, University of Aveiro, Aveiro 3810-193, Portugal \\ ${ }^{\mathrm{c}}$ CIQ/Department of Chemistry and Biochemistry, Faculty of Sciences, University of Porto, Porto 4169-007, Portugal \\ ${ }^{\mathrm{d}}$ Department of Chemical Engineering, School of Engineering (ISEP), Polytechnic Institute of Porto, Porto 4200-072, Portugal
}

\section{Keywords:}

Alkyl hydroxycinnamates

Antioxidant activity Caffeic

acid

Ferulic acid Sinapic

acid

Drug-likeness properties

\section{a b s t r a c t}

Long chain alkyl hydroxycinnamates (8e21) were synthesized from the corresponding half esters of malonic acid (5e7) and benzaldehyde derivatives by Knoevenagel condensation. The total antioxidant capacity of these hydroxycinnamyl esters was evaluated using DPPH and ABTS assays. The observed antioxidant activity was highest for esters of caffeic acid followed by sinapic esters and ferulic esters. The parameters for drug-likeness of these hydroxycinnamyl esters were also evaluated according to the Lipinski's 'rule-of-five'. All the ester derivatives were found to violate one of the Lipinski's parameters ( $\operatorname{LLg} \mathrm{P}>5$ ), even though they have been found to be soluble in protic solvents. The predictive topological

polar surface area (TPSA) data allow concluding that they could have a good capacity for penetrating cell

membranes. Therefore, one can propose these novel lipophilic compounds as potential antioxidants for tackling oxidative processes.

\section{Introduction}

Phenolic acids, especially hydroxycinnamic acids, such as $p$-coumaric, ferulic, caffeic and sinapic acids, are natural hydrophilic antioxidants which occur in fruits, vegetables, spices and herbs. Hydroxyl groups present on cinnamic acids are often associated with their antioxidant activity. [1]. Hydroxycinnamic acids are of particular interestbecause of their potential biological outline dis- playing antioxidant, chelating, freeradical scavenging, antiallergic, anti-inflammatory, antimicrobial, antiviral, anticarcinogenic and UV filter properties [1]. However these types of phenolic acids have relatively low solubility in aprotic media. The hydrophilic character of these antioxidants reduces their effectiveness in stabilizing lipophilic systems, such as fats and oils and has been reported as a serious disadvantage if an aqueous phase is also present. The hydrophobicity of phenolic acids can be enhanced by chemical esterification of the carboxyl group of the phenolic acid with a fatty alcohol to obtain an amphiphilic molecule, which should keep its original functional properties and therefore, extend the putative applications of these natural antioxidants in oil based food

\footnotetext{
* Corresponding authors.

E-mail addresses: shrivkamat@yahoo.com (S.P. Kamat), fborges@fc.up.pt (F. Borges).
}

processing and cosmetics. [1]. Long chain alkyl cinnamates isolated from Sophora flavescens have been shown to be free radical scav- engers [2]. The antioxidant activity of radish sprout (Raphanus sativus $\mathrm{L}$ ) has been attributed to be related mainly with the presence of sinapic acid esters [3]. Similarly, esters of long chain alcohols with caffeic acid, isolated from Hypericum laricifolium have been shown to inhibit COX-1 enzymes [4]. Esters of sinapic and ferulic acids with alkanols have been also used in commercial applications as sunscreens [5,6]. Recently, the occurrence of a relationship between the redox potentials and the antioxidant activity of hydroxycin- namic acids and derivatives has been demonstrated [7].

In this context, this work reports the design and synthesis of anewset of synthetic long chain alkyl cinnamyl esters using natural cinnamic acids as templates. In addition, the antioxidant activity of the hydroxylated long chain alkyl cinnamates and their precursors was studied along with the evaluation oftheirdrug-likeness profile.

\section{Results and discussions}

\subsection{Chemistry}

Long chain alkyl cinnamates have been previously prepared by using the appropriate cinnamic acid $[8,9]$ or acid chloride $[10]$ and the suitable long chain alkanol. This type of study was only 
conducted for identification purposes of the corresponding natural cinnamyl esters. However the synthetic procedures reported so far involve protection and deprotection steps, operations that conduct in general to low to moderate yields $[8,9]$. So, herein a novel synthetic strategy is presented in which the synthesis of long chain alkyl cinnamates $8 \mathrm{e} 21$ was performed by using monomalonates $5 \mathrm{e} 7$ and substituted benzaldehyde derivatives as starting material and applying the Verley-Doebner modification of Knoevenagel condensation reaction[11,12] (Scheme1).

Monomalonates were obtained in high yields by simply heating Meldrum's acid (2, 2-dimethyl-1, 3-dioxane-4, 6-dione) 4 with alcohols in toluene $[11,13]$.The half esters of malonic acid $5 \mathrm{e} 7$ were obtained by refluxing tetradecanol (1), hexadecanol (2) and octa- decanol (3) with Meldrum's acid 4. Half esters of malonic acid 5, 6 and 7 are new compoundsand were fully characterized on the basis

of their spectral data (FT-IR, ${ }^{1} \mathrm{H} \&{ }^{13} \mathrm{C}$ NMR and HRESIMS).

Further condensation of monomalonates $5 \mathrm{e} 7$ with protocatechualdehyde, vanillin, syringaldehyde, 4-hydroxybenzaldehyde, 4methoxybenzaldehyde, veratraldehyde, and benzaldehyde in the presence of dry pyridine and b-alanine was performed through a Verley-Doebner modification of Knoevenagel condensation [12] giving alkyl cinnamates (8e21) (see Table 1) in good yields ranging from $85 \%$ to quantitative. The long chainalkyl cinnamates 9,12 and 14 arenewcompounds, characterizedby detailspectraldata(FT-IR, ${ }_{1}^{1} \mathrm{H} \&{ }^{13} \mathrm{C}$ NMR and HRESIMS) and reported in the experimental section whereas the known compounds $(8,10,11,13$, 15e21) were charac- terized by spectral data and compared with literature reports.

\subsection{Evaluation of the total antioxidant capacity (TAC) of the cinnamic derivatives}

Total antioxidant capacity (TAC) assays have been often used to determine the hierarchy of radical-scavenging abilities of potential phenolic antioxidant compounds that work either through electron- or H- donating mechanisms [14,15]. Accordingly, DPPH $\left(2,2^{0}\right.$-diphenyl-1-picrylhydrazyl radical $)$ and $\mathrm{ABTS}^{\mathrm{b}}\left(2,2^{0}\right.$-azinobisethylbenzothiazoline-6-sulfonic acid)) assays were used to assess the radical-scavenging ability of hydroxycinnamic ester derivatives (Table 2). Out of the 10 hydroxycinnamic esters only compounds 8,10 , $11,12,13,18,19$ and 20 were evaluated for antioxidant activity since esters 15 and 16 were isolated from natural sources and have been evaluated earlier [2]. The TEAC values of the compounds under study obtained from the ABTS ${ }^{\mathrm{b}}$ decolourization assay are presented in Table2.Theradical-scav-

enging ability was only determined for the most outstanding antioxidants (based on DPPH data). Trolox, the water-soluble vitamin $E$ analogue, was used as standard.

The radical-scavenging ability data obtained for hydroxycin- namic esters against DPPH were in good agreement with the expected activities of this type of phenolic systems: it is higher when a catechol group is present (caffeic series), and lower when the meta-hydroxyl function is substituted by a methoxyl group (ferulic series), having the sinapic series intermediate values
Table 1

Long chain alkyl cinnamyl esters (8e21).<smiles>[R]OC(=O)/C=C/c1cc([R2])c([R2])c([R2])c1</smiles>

\begin{tabular}{|c|c|c|c|c|c|c|}
\hline Cinnamates & $\mathrm{R}_{1}$ & $\mathrm{R}_{2}$ & $\mathrm{R}_{3}$ & $\mathrm{R}_{4}$ & Yıеıd \% & Reference \\
\hline$\overline{88}$ & $\overline{\mathrm{C}_{14} \mathrm{H}_{29}}$ & $\overline{\mathrm{H}}$ & $\overline{\mathrm{OH}}$ & $\overline{\mathrm{H}}$ & $\overline{95}$ & [9] \\
\hline 9 & $\mathrm{C}_{14} \mathrm{H}_{29}$ & $\mathrm{H}$ & $\mathrm{OCH}_{3}$ & $\mathrm{H}$ & 95 & $\mathrm{~N}^{\#}$ \\
\hline 10 & $\mathrm{C}_{14} \mathrm{H}_{29}$ & $\mathrm{OH}$ & $\mathrm{OH}$ & $\mathrm{H}$ & 91 & [9] \\
\hline 11 & $\mathrm{C}_{14} \mathrm{H}_{29}$ & $\mathrm{OCH}_{3}$ & $\mathrm{OH}$ & $\mathrm{H}$ & Quant.\# & [25] \\
\hline 12 & $\mathrm{C}_{14} \mathrm{H}_{29}$ & $\mathrm{OCH}_{3}$ & $\mathrm{OH}$ & $\mathrm{OCH}_{3}$ & 85 & $\mathrm{~N}$ \\
\hline 13 & $\mathrm{C}_{16} \mathrm{H}_{33}$ & $\mathrm{OH}$ & $\mathrm{OH}$ & $\mathrm{H}$ & Quant. & [24] \\
\hline 14 & $\mathrm{C}_{16} \mathrm{H}_{33}$ & $\mathrm{OCH}_{3}$ & $\mathrm{OCH}_{3}$ & $\mathrm{H}$ & 88 & $\mathrm{~N}$ \\
\hline 15 & $\mathrm{C}_{16} \mathrm{H}_{33}$ & $\mathrm{OCH}_{3}$ & $\mathrm{OH}$ & $\mathrm{H}$ & Quant. & [2] \\
\hline 16 & $\mathrm{C}_{16} \mathrm{H}_{33}$ & $\mathrm{OCH}_{3}$ & $\mathrm{OH}$ & $\mathrm{OCH}_{3}$ & 94 & [2] \\
\hline 17 & $\mathrm{C}_{16} \mathrm{H}_{33}$ & $\mathrm{H}$ & $\mathrm{H}$ & $\mathrm{H}$ & 95 & [27] \\
\hline 18 & $\mathrm{C}_{18} \mathrm{H}_{37}$ & $\mathrm{OH}$ & $\mathrm{OH}$ & $\mathrm{H}$ & 89 & [24] \\
\hline 19 & $\mathrm{C}_{18} \mathrm{H}_{37}$ & $\mathrm{OCH}_{3}$ & $\mathrm{OH}$ & $\mathrm{H}$ & 97 & [28] \\
\hline 20 & $\mathrm{C}_{18} \mathrm{H}_{37}$ & $\mathrm{OCH}_{3}$ & $\mathrm{OH}$ & $\mathrm{OCH}_{3}$ & 93 & [29] \\
\hline 21 & $\mathrm{C}_{18} \mathrm{H}_{37}$ & $\mathrm{H}$ & $\mathrm{H}$ & $\mathrm{H}$ & 95 & [29] \\
\hline
\end{tabular}

\# Quant.-Quantitative, N-New.

(Table 2) $[7,16]$. In the same series the lipophilic esters exhibit approximately the sameantioxidantperformance.

According to the data obtained in the ABTS assay, only caffeic acid and its esters disclose an antioxidant activity similar to that presented by Trolox. The results obtained are in good agreement with the expected activities of this type of phenolic systems since it was described that the ability of an antioxidant to scavenge the

artificial long-lived radical monocation ABTS $\quad \mathbf{b}$ depend on the resonancestabilization energyofthephenoxylradicalspecies [16].

The antiradical properties obtained for the known hydroxycin- namic derivatives $(8,10)$ compare well with published values $[9,16 \mathrm{e} 19]$. Moreover, the ABTS results follow the same tendency, although not as noticeable, verified from the results obtained with the DPPH method.

\subsection{Calculation of drug-likeness properties}

Drug-likeness can be deduced as a delicate balance among the molecular properties of a compound that directly influence its pharmacodynamics and pharmacokinetics and ultimately affect their absorption, distribution, metabolism, and excretion in human body like a drug [20]. In general, these parameters allow to ascertain a poor oral absorption, or membrane permeability, that occurs when the evaluated molecules present values higher than five H-bond donors (HBD), $10 \mathrm{H}$ bond acceptors (HBA), molecular weight (MW) > 500 Da and LogP (cLogP) $>5$ (Lipinski's 'rule-of-five') [21]. 
<smiles>[R]O[IH+][IH+]</smiles>

1-3
4 $\stackrel{\text { (i ) } \longrightarrow}{\longrightarrow} \prod_{\mathrm{O}}^{-\mathrm{O}} \prod_{\mathrm{O}-7}^{\mathrm{O}}{ }_{-\mathrm{H}}^{\text {(ii) }}$

$5-7$
8-21

(i) Toluene, reflux, $4 \mathrm{hr}$ (ii) benzaldehyde derivatives, pyridine, $\beta$-alanine

Scheme 1. Synthesis of long chain alkyl cinnamates $8 \mathrm{e} 21$ from monomalonates $5 \mathrm{e} 7$.

Table 2

Structural properties of the phenolic acids and derivatives under study. ${ }^{\mathrm{a}}$

\begin{tabular}{|c|c|c|c|c|c|c|c|c|c|}
\hline TPSA & DPPH IC $50(\mathrm{~mm})$ & ABTS ${ }^{\mathrm{pb}}$ & Molecular weight & $\operatorname{miLog} P^{\mathrm{a}}$ & n-ROTB & $n$-ON acceptors & $n$-ONNH donors & Volume & TPSA \\
\hline p-coumaric acid & $>100$ & $\mathrm{e}^{\mathrm{c}}$ & 164.16 & 1.43 & 2 & 3 & 2 & 146.48 & 57.53 \\
\hline 8 & $>100$ & $\mathrm{e}^{\mathrm{c}}$ & 360.54 & 8.43 & 16 & 3 & 1 & 382.43 & 46.53 \\
\hline Caffeic acid & 34.1 & 0.95 & 180.16 & 0.94 & 2 & 4 & 3 & 154.50 & 77.76 \\
\hline 10 & 31.9 & 0.98 & 376.54 & 8.05 & 16 & 4 & 2 & 390.45 & 66.76 \\
\hline 13 & 33.0 & 0.96 & 404.59 & 8.72 & 18 & 4 & 2 & 424.05 & 66.76 \\
\hline 18 & 38.0 & 1.02 & 432.64 & 9.12 & 20 & 4 & 2 & 457.66 & 66.76 \\
\hline Ferulic acid & 111.6 & 1.76 & 194.19 & 1.25 & 3 & 4 & 2 & 172.02 & 66.76 \\
\hline 11 & 110.2 & 1.66 & 390.56 & 8.30 & 17 & 4 & 1 & 407.97 & 55.77 \\
\hline 19 & 117.0 & 1.80 & 446.67 & 9.22 & 21 & 4 & 1 & 475.18 & 55.77 \\
\hline Sinapic acid & 66.0 & 1.14 & 224.21 & 1.26 & 4 & 5 & 2 & 197.57 & 75.99 \\
\hline 12 & 77.2 & 1.18 & 420.59 & 8.32 & 18 & 5 & 1 & 433.52 & 65.00 \\
\hline 20 & 77.1 & 1.16 & 476.70 & 9.22 & 22 & 5 & 1 & 500.73 & 65.00 \\
\hline
\end{tabular}

${ }^{\mathrm{a}} \mathrm{n}$-ROTB, number of rotatable bonds; $\mathrm{n}-\mathrm{OHNH}$, number of hydrogen bond donors; $\mathrm{n}$-ON, number of hydrogen acceptors; TPSA, topological polar surface area

b mmol AH equivalent to 1 mmol Trolox.

${ }^{\mathrm{c}}$ not determined.

However, it is important to note that there are many violations of this rule among existing drugs and vice versa, and therefore, qualifying the "rule of five" does not guarantee that a molecule is "drug-like" [20]. Topological polar surface area (TPSA) is now been recognized as a good indicator of drug absorbance in the intestines, Caco- 2 monolayers penetration, and bloodebrainbarriercrossing [22].

The mentioned parameters were calculated for the hydroxycin- namic esters in analysis and the results are depicted in Table 2. From the data obtained, onecannoticethat the phenolicacidsarenotableto cross membranes effectively, once they have a $\log D \quad 1$. The alkyl cinnamic esters possess an adequate number of proton acceptor and proton donor groups to ensure efficient interaction with the hydrogen- bonding groups of the receptors. Hydrogen-bonding capacity has been also identified as an important parameter for describing drug permeability $[20,21]$. All the ester derivatives were found to violate one of the Lipinski's parameters $(\log \mathrm{P}(\mathrm{cLogP})>5)$, althoughtheyhave

been found to have solubility in protic solvents. Accordingly to their predictive TPSA data it seems that this type of cinnamic esters could have a good capacity for penetrating cellmembranes.

\section{Experimentalsection}

\subsection{General}

Meldrum's acid was prepared according to literature procedure [23]. Melting points were recorded using Thiele tube and are uncor- rected. UV spectrawererecordedonaShimadzuUV-2450UVeVisible spectrophotometer. IR spectra were recorded as $\mathrm{KBr}$ diluted pellets on a Shimadzu (IR Prestige-21) FTIR spectrophotometer. ${ }^{1} \mathrm{H}$ and ${ }^{13} \mathrm{C}$ NMR spectra were recorded at 300 and $75 \mathrm{MHz}$, respectively on a Bruker

WT 300 FT-NMR instrument with TMS as internal standard. Chemical shifts are recorded in $\mathrm{d}$ values and coupling constant () are given in $\mathrm{Hz}$. The multiplicities of carbon signals were obtained from dis- tortionless enhancement by polarization transfer (DEPT). High reso- lution mass spectra (HRMS-ESIp) were performed on a microTOF (focus) mass spectrometer. Ions were generated using an ApolloII (ESI) source. Ionization was achieved by electrospray, using a voltage of $4500 \mathrm{~V}$ applied to the needle, and a counter voltage between 100 and $150 \mathrm{~V}$ applied to the capillary.Allyieldsrefertoisolatedproducts unlessstatedotherwise.

\subsection{Synthesis of half esters of malonic acid (5e7)}

Equimolar quantities $(10 \mathrm{mmol})$ of appropriate alcohols $1 \mathrm{e} 3$ and Meldrum's acid 4 were refluxed in toluene $(5 \mathrm{~mL})$ for $4 \mathrm{~h}$. The reaction was cooled to room temperature and then extracted with saturated $\mathrm{NaHCO}_{3}$ solution. The bicarbonate layer was neutralized with conc. $\mathrm{HCl}$ to release the half esters of malonic acid, which were extracted with diethyl ether, washed with water, dried over anhy-

drous $\mathrm{Na}_{2} \mathrm{SO}_{4}$ and evaporated to yield pure half esters of malonic acid $5 \mathrm{e} 7$.

\subsubsection{Monotetradecyl malonate (5)}

Colorless solid; yield 95\%; m.p. 45 C (Petroleum ether); IR (KBr, $\mathrm{cm}^{1}$ ): 3225e3500, 2918,1744 (ester C0),1692 (acid C0),1284,1166, 825; ${ }^{1}$ HNMR(300 $\left.\mathrm{MHz}, \mathrm{CDCl}_{3}\right) \mathrm{d}: 0.88\left(\mathrm{t}, \mathrm{J}^{1 / 4} 6.6 \mathrm{~Hz}, 3 \mathrm{H}, \mathrm{H}-14^{0}\right), 1.26$ (bs, $\left.22 \mathrm{H}, \mathrm{H}-3^{0}-13^{0}\right), 1.65$ (m, 2H, H-2 ${ }^{0}$ ), 3.43 (s, 2H, H-2), 4.17 $\left(\mathrm{t}, J^{1 / 4} 6.6 \mathrm{~Hz}, 2 \mathrm{H}, \mathrm{H}-1^{0}\right) ;{ }^{13} \mathrm{CNMR}\left(75 \mathrm{MHz}, \mathrm{CDCl}_{3}\right) \mathrm{d}: 14.1\left(\mathrm{C}-14^{0}\right), 22.7\left(\mathrm{C}-13^{0}\right)$, $25.8\left(\mathrm{C}^{0} 3^{0}\right), 28.4\left(\mathrm{C}-2^{0}\right), 29.3\left(\mathrm{C}-4^{0}, 11^{0}\right), 29.6\left(\mathrm{C}-5^{0}-10^{0}\right), 31.9$ $\left(\mathrm{C}-12^{0}\right), 40.5(\mathrm{C}-2), 65.7\left(\mathrm{C}-1^{0}\right), 167.3(\mathrm{C}-3), 170.6(\mathrm{C}-1)$;HRESIMS: $\mathrm{m} / \mathrm{z}$ $323.2195(\mathrm{M} \mathrm{p} \mathrm{Na})^{\text {; }}$; Calcd for $\mathrm{C}_{17} \mathrm{H}_{32} \mathrm{O}_{4} \mathrm{Na}^{\mathrm{b}}: 323.2198$.

\subsubsection{Monohexadecyl malonate (6)}

Colorless solid; yield 90\%; m.p. 59 C (Petroleum ether); IR (KBr, $\mathrm{cm}^{1}{ }^{1}$ ): 2400e3600, 1744 (ester CO), 1695 (acid CO), 1283, 1166, 750; ${ }^{1}$ H NMR (300 $\left.\mathrm{MHz}, \mathrm{CDCl}_{3}\right) \mathrm{d}: 0.88\left(\mathrm{t}, J 1 / 46.6 \mathrm{~Hz}, 3 \mathrm{H}, \mathrm{H}-16^{0}\right), 1.26(\mathrm{bs}$, $\left.26 \mathrm{H}, \mathrm{H}-3^{0}-15^{0}\right), 1.67\left(\mathrm{~m}, 2 \mathrm{H}, \mathrm{H}-2^{0}\right), 3.45(\mathrm{~s}, 2 \mathrm{H}, \mathrm{H}-2), 4.20\left(\mathrm{t}, J^{1} / 46.6 \mathrm{~Hz}\right.$, $\left.2 \mathrm{H}, \mathrm{H}-1^{0}\right) ;{ }^{13} \mathrm{CNMR}\left(75 \mathrm{MHz}, \mathrm{CDCl}_{3}\right) \mathrm{d}: 14.1\left(\mathrm{C}-16^{0}\right), 22.7\left(\mathrm{C}-15^{0}\right), 25.7$ $\left(\mathrm{C}-3^{0}\right), 28.4\left(\mathrm{C}-2^{0}\right), 29.3\left(\mathrm{C}-4^{0}, 13^{0}\right), 29.7\left(\mathrm{C}-5^{0}-12^{0}\right), 31.9\left(\mathrm{C}-14^{0}\right), 40.6$ (C-2), $65.7\left(\mathrm{C}-1^{0}\right), 167.2$ (C-3), 170.9 (C-1); HRESIMS: $m / z$ 351.2516 $(\mathrm{MpNa})^{\mathrm{b}}$;CalcdforC ${ }_{19} \mathrm{H}_{36} \mathrm{O}_{4} \mathrm{Na}^{\mathrm{b}}: 351.2511$.

\subsubsection{Monooctadecyl malonate (7)}

Colorless solid; yield 96\%; m.p. $65 \mathrm{C}$ (Petroleum ether); $\mathrm{IR} \mathrm{(KBr,} \mathrm{cm}^{1}$ ): 2400e3600, 1745 (ester C0), 1693(acid CO), 1284, 1166, 750; ${ }^{1}$ H NMR (300 $\left.\mathrm{MHz}, \mathrm{CDCl}_{3}\right) \mathrm{d}: 0.88\left(\mathrm{t}, J^{1 / 4} 6.6 \mathrm{~Hz}, 3 \mathrm{H}, \mathrm{H}-18^{0}\right), 1.26(\mathrm{bs}$,

$\left.30 \mathrm{H}, \mathrm{H}-3^{0}-17^{0}\right), 1.69\left(\mathrm{~m}, 2 \mathrm{H}, \mathrm{H}-2^{0}\right), 3.44(\mathrm{~s}, 2 \mathrm{H}, \mathrm{H}-2), 4.19(\mathrm{t},)^{1 / 4} 6.6 \mathrm{~Hz}$, $\left.2 \mathrm{H}, \mathrm{H}-1^{0}\right) ;{ }^{13} \mathrm{CNMR}\left(75 \mathrm{MHz}, \mathrm{CDCl}_{3}\right) \mathrm{d}: 14.1\left(\mathrm{C}-18^{0}\right), 22.7\left(\mathrm{C}-17^{0}\right), 25.7$ $\left(\mathrm{C}-3^{0}\right), 28.4\left(\mathrm{C}-2^{0}\right), 29.3\left(\mathrm{C}-4^{0}, 15^{0}\right), 29.7\left(\mathrm{C}-5^{0}-14^{0}\right), 31.9\left(\mathrm{C}-16^{0}\right), 40.5$ (C-2), $65.7\left(\mathrm{C}-1^{0}\right), 167.3(\mathrm{C}-3), 170.3$ (C-1); HRESIMS: $m / z$ 379.2827 $(\mathrm{MpNa})^{\mathrm{p}}$;CalcdforC $\mathrm{C}_{21} \mathrm{H}_{40} \mathrm{O}_{4} \mathrm{Na}^{\mathrm{b}}: 379.2824$. 


\subsection{Synthesis of long chain alkyl cinnamates (8e21)}

Equimolar quantities ( $2 \mathrm{mmol}$ ) of the appropriate half ester of malonic acid and the appropriate benzaldehyde derivative along with dry pyridine $(1.0 \mathrm{~mL})$ and $\mathrm{b}$-alanine $(12 \mathrm{e} 15 \mathrm{mg})$ were refluxed for $90 \mathrm{e} 110 \mathrm{~min}$. For hydroxybenzaldehydes the reaction mixtures were kept at room temperature in Erlenmeyer flasks with loosely fitted bar corks for 2 weeks. Reaction mixture was cooled in an ice bath and conc. $\mathrm{HCl}(1.0 \mathrm{~mL})$ was added. Extraction with diethyl ether or filtration on a Buchner funnel afforded the crude cinna- mates, 8e21 which were further purified by recrystallization.

\subsubsection{Trans-tetradecyl-3-(4-hydroxyphenyl) propenoate (8)}

Colorless solid; m.p. 89 C (methanol) (lit. [9] 88e90 C); HRE- SIMS: $m / z$ 383.2554 (M p Na) ${ }^{\text {p; }}$ Calcd for $\mathrm{C}_{23} \mathrm{H}_{36} \mathrm{O}_{3} \mathrm{Na}^{\text {p }}$ : 383.2557 .

Identical with ref [9].

\subsubsection{Trans-tetradecyl-3-(4-methoxyphenyl) propenoate (9)}

Colorless solid; m.p. $51 \mathrm{C}$ (methanol); UV (MeOH): 309, $226 \mathrm{~nm}$; IR $\left(\mathrm{KBr}, \mathrm{cm}^{1}\right.$ ): 2916, 1707 (CO), 1606, 1517, 1182, 825; ${ }^{1} \mathrm{H}$ NMR $(300 \mathrm{MHz}$, $\mathrm{CDCl}_{3}$ ) d: $0.88\left(\mathrm{t}, J 1 / 46.6 \mathrm{~Hz}, 3 \mathrm{H}, \mathrm{H}-14^{0}\right), 1.26(\mathrm{bs}$,

$\left.22 \mathrm{H}, \mathrm{H}-3^{0}-13^{0}\right), 1.69\left(\mathrm{~m}, 2 \mathrm{H}, \mathrm{H}-2^{0}\right), 3.84\left(\mathrm{~s}, 3 \mathrm{H}, \mathrm{OCH}_{3}\right), 4.18$

(t, J $1 / 46.6 \mathrm{~Hz}, 2 \mathrm{H}, \mathrm{H}-1^{0}$ ), 6.31 (d, J $\left.1 / 415.9 \mathrm{~Hz}, 1 \mathrm{H}, \mathrm{H}-2\right), 6 . \overline{9} 0$

(d, J 1/4 $8.8 \mathrm{~Hz}, 2 \mathrm{H}, \mathrm{H}-6,8), 7.48$ (d, J $1 / 48.8 \mathrm{~Hz}, 2 \mathrm{H}, \mathrm{H}-5,9$ ), 7.64 (d, J 1/4

$15.9 \mathrm{~Hz}, 1 \mathrm{H}, \mathrm{H}-3$ ); HRESIMS: $m / z \quad 397.2704(\mathrm{M} \mathrm{p} \mathrm{Na})^{\mathrm{p}}$; Calcd for $\mathrm{C}_{24} \mathrm{H}_{38} \mathrm{O}_{3} \mathrm{Na}^{\text {p: }} 397.2713$.

3.3.3. Trans-tetradecyl-3-(3,4-dihydroxyphenyl)propenoate (10)

Colorless solid; m.p. 106 C (methanol) (lit [9,24]. 108e109 C); HRESIMS: $m / z \quad 399.2508(\mathrm{M} \mathrm{p} \mathrm{Na})^{\mathrm{p}}$; Calcd for $\mathrm{C}_{23} \mathrm{H}_{36} \mathrm{O}_{4} \mathrm{Na}^{\mathrm{p}}$ : 399.2506 . Identical with ref [9] and [24].

\subsubsection{Trans-tetradecyl-3-(4-hydroxy-3-methoxyphenyl)}

propenoate (11)

Colorless solid; m.p. 70 C (methanol) (lit [25]. 76 C); identical with ref [25].

\subsubsection{Trans-tetradecyl-3-(3,5-dimethoxy-4-hydroxyphenyl)}

propenoate (12)

Colorless solid;m.p.62 C (methanol);UV(MeOH):327,240nm; IR (KBr,cm $\left.{ }^{1}\right): 3554(\mathrm{OH}), 2917,1709(\mathrm{CO}), 1639,1516,1181,843 ;{ }^{1}{ }^{\mathrm{HNMR}}\left(300 \mathrm{MHz}, \mathrm{CDCl}_{3}\right) \mathrm{d}$ : $0.87\left(\mathrm{t}, \mathrm{J}^{1} / 46.6 \mathrm{~Hz}, 3 \mathrm{H}, \mathrm{H}-14^{0}\right), 1.25\left(\mathrm{bs}, 22 \mathrm{H}, \mathrm{H}-3^{0}\right.$ -

$\left.13^{0}\right), 1.69\left(\mathrm{~m}, 2 \mathrm{H}, \mathrm{H}-2^{0}\right), 3.91\left(\mathrm{~s}, 6 \mathrm{H}, 2 \quad \mathrm{OCH}_{3}\right), 4.18(\mathrm{t},]^{1 / 4} 6.6 \mathrm{~Hz}, 2 \mathrm{H}, \mathrm{H}-$ $\left.1^{0}\right), 5.81(\mathrm{~s}, 1 \mathrm{H}, \mathrm{OH}), 6.30\left(\mathrm{~d}, J \frac{1}{4} 15.9 \mathrm{~Hz}, 1 \mathrm{H}, \mathrm{H}-\overline{2}\right), 6.77(\mathrm{~s}, 2 \mathrm{H}, \mathrm{H}-5,9)$, 7.58(d, J1/4 15.9Hz,1H,H-3); ${ }^{13} \mathrm{CNMR}\left(75 \mathrm{MHz}^{1} \mathrm{CDCl}_{3}\right) \mathrm{d}: 14.1\left(\mathrm{C}-14^{0}\right)$, $22.7\left(\mathrm{C}-13^{0}\right), 25.9\left(\mathrm{C}-3^{0}\right), 28.8\left(\mathrm{C}-2^{0}\right), 29.2\left(\mathrm{C}-4^{0}\right), 29.3\left(\mathrm{C}-11^{0}\right), 29.6\left(\mathrm{C}-5^{0}-\right.$ $\left.10^{0}\right), 31.9\left(\mathrm{C}-12^{0}\right), 56.3\left(2 \mathrm{OCH}_{3}\right), 64.6\left(\mathrm{C}-1^{0}\right), 105.1(\mathrm{C}-5,9), 116.1(\mathrm{C}-2)$, 126.0 (C-4), 137.1 (C-7), 144.8 (C-3), 147.2 (C-6, 8), 167.2 (C-1); HRE- SIMS: $m / z$ $443.2762(\mathrm{M} \mathrm{p} \mathrm{Na})^{\mathrm{p}}$; Calcd for $\mathrm{C}_{25} \mathrm{H}_{40} \mathrm{O}_{5} \mathrm{Na}^{\mathrm{p}}$ : 443.2768 .

3.3.6. 3.3Trans-hexadecyl-3-(3,4-dihydroxyphenyl)propenoate (13) Colorless solid; m.p.102 C (methanol) (lit [24].110e111 C); UV (MeOH): 329, $219 \mathrm{~nm}$; IR (KBr, cm ${ }^{1}$ ): $3481(\mathrm{OH}), 3312(\mathrm{OH}), 2918$, 1684(CO),1606,1286,1180,974,815; ${ }^{1} \mathrm{HNMR}\left(300 \mathrm{MHz}, \mathrm{CDCl}_{3}\right) \mathrm{d}$ : $0.87\left(\mathrm{t}, J^{1} / 46.6 \mathrm{~Hz}, 3 \mathrm{H}, \mathrm{H}-16^{0}\right), 1.25\left(\mathrm{bs}, 26 \mathrm{H}, \mathrm{H}-3^{0}-15^{0}\right), 1.67(\mathrm{~m}, 2 \mathrm{H}$, $\mathrm{H}-2^{0}$ ), $4.18\left(\mathrm{t}, J^{1 / 4} 6.75 \mathrm{~Hz}, 2 \mathrm{H}, \mathrm{H}-1^{0}\right.$ ), 5.69 (bs, 2H, $\left.2 \mathrm{OH}\right), 6.27$ (d, J1/4 15.9Hz,1H,H-2), 6.87(d, J1/4 8.1Hz,1H,H-8),7.02(dd, J1/48.1, $1.8 \mathrm{~Hz}, 1 \mathrm{H}, \mathrm{H}-9), 7.09\left(\mathrm{~d}, J^{1 / 4} 1.8 \mathrm{~Hz}, 1 \mathrm{H}, \mathrm{H}-5\right), 7.57\left(\mathrm{~d}, J^{1 / 4} 15.9 \mathrm{~Hz}, 1 \mathrm{H}\right.$, H-3); ${ }^{13}$ CNMR(75 MHz,DMSO-d 6 ) d: $13.4\left(\mathrm{C}-16^{0}\right), 21.6\left(\mathrm{C}-15^{0}\right), 24.9$ $\left(\mathrm{C}-3^{0}\right), 27.7\left(\mathrm{C}-2^{0}\right), 28.1\left(\mathrm{C}-4^{0}, 13^{0}\right), 28.5\left(\mathrm{C}-5^{0}-12^{0}\right), 30.8\left(\mathrm{C}-14^{0}\right), 63.2$ $\left(\mathrm{C}-1^{0}\right), 113.5(\mathrm{C}-2), 114.3(\mathrm{C}-8), 115.2(\mathrm{C}-5), 120.8(\mathrm{C}-9), 125.0(\mathrm{C}-4)$, 144.5 (C-6), 145.1 (C-3), 147.9 (C-7), 166.1 (C-1); HRESIMS: $m / z$ $427.2815(\mathrm{M} \mathrm{p} \mathrm{Na})^{\text {p }}$; Calcd for $\mathrm{C}_{25} \mathrm{H}_{40} \mathrm{O}_{4} \mathrm{Na}^{\text {p }}$ : 427.2819 .

\subsubsection{Trans-hexadecyl-3-(3,4-dimethoxyphenyl) propenoate (14)}

Colorless solid; m.p. 67 C (methanol); UV (MeOH): 322, 295, 235, 218 $\mathrm{nm}$; IR(KBr, $\mathrm{cm}^{1}$ ): 2916,1720(CO),1514,1271,976,843;

${ }^{1} \mathrm{HNMR}\left(300 \mathrm{MHz}^{\mathrm{CDCl}}{ }_{3}\right) \mathrm{d}: 0.87\left(\mathrm{t}, J 1 / 46.6 \mathrm{~Hz}, 3 \mathrm{H}, \mathrm{H}-16^{0}\right), 1.25(\mathrm{bs}$, $\left.26 \mathrm{H}, \mathrm{H}-3^{0}-15^{0}\right), 1.69\left(\mathrm{~m}, 2 \mathrm{H}, \mathrm{H}-2^{0}\right), 3.91\left(\mathrm{~s}, 6 \mathrm{H}, 2 \quad \mathrm{OCH}_{3}\right), 4.18$ (t, J $1 / 46.6 \mathrm{~Hz}, 2 \mathrm{H}, \mathrm{H}-1^{0}$ ), 6.31 (d, J $\left.1 / 415.9 \mathrm{~Hz}, 1 \mathrm{H}, \mathrm{H}-2\right), 6 . \overline{86}$ (d, $J 1 / 48.4 \mathrm{~Hz}, 1 \mathrm{H}, \mathrm{H}-8), 7.05(\mathrm{~d}, J 1 / 41.8 \mathrm{~Hz}, 1 \mathrm{H}, \mathrm{H}-5), 7.10\left(\mathrm{dd}, J^{1 / 4} 48.1\right.$, $1.8 \mathrm{~Hz}, 1 \mathrm{H}, \mathrm{H}-9$ ), 7.62 (d, J 1/4 $15.9 \mathrm{~Hz}, 1 \mathrm{H}, \mathrm{H}-3$ ); HRESIMS: $m / z$ 455.3149 (M p Na) $^{\text {p }}$; Calcd for $\mathrm{C}_{27} \mathrm{H}_{44} \mathrm{O}_{4} \mathrm{Na}^{\mathrm{p}}$ : 455.3132 .

3.3.8. Trans-hexadecyl-3-(4-hydroxy-3-methoxyphenyl) propenoate (15)

Colorless solid; m.p. 69 C (methanol) (lit [26,2]. 61e63 C); identical with ref [26] and [2].

\subsubsection{Trans-hexadecyl-3-(3,5-dimethoxy-4-hydroxyphenyl)} propenoate (16)

Colorless solid; m.p. $60 \mathrm{C}$ (methanol); HRESIMS: $m / z$ 471.3078 (M p $\mathrm{Na})^{\text {p }}$; Calcd for $\mathrm{C}_{27} \mathrm{H}_{44} \mathrm{O}_{5} \mathrm{Na}^{\mathrm{p}}$ : 471.3081. Identical with ref [2].

\subsubsection{Trans-hexadecyl-3-phenylpropenoate (17)}

Colorless solid; m.p. 38 C (Petroleum ether) (lit [27]. 39.6e40.6 C); UV (MeOH): 276, $217 \mathrm{~nm}$; IR (KBr, $\mathrm{cm}^{1}$ ): 2924,

1717 (CO), 1638,1310,1167,766; ${ }^{1} \mathrm{HNMR}\left(300 \mathrm{MHz}^{\mathrm{C}} \mathrm{CDCl}_{3}\right) \mathrm{d}: 0.88\left(\mathrm{t}, J^{1 / 4} 6.6\right.$ $\left.\mathrm{Hz}, 3 \mathrm{H}, \mathrm{H}-16^{0}\right), 1.25\left(\mathrm{bs}, 26 \mathrm{H}, \mathrm{H}-3^{0}-15^{0}\right), 1.69\left(\mathrm{~m}, 2 \mathrm{H}, \mathrm{H}-2^{0}\right)$, $4.20\left(\mathrm{t}, J^{1 / 4} 6.6 \mathrm{~Hz}, 2 \mathrm{H}, \mathrm{H}-1^{0}\right), 6.44\left(\mathrm{~d}, J^{1 / 4} 15.9 \mathrm{~Hz}, 1 \mathrm{H}, \mathrm{H}-2\right), 7.37 \mathrm{e} 7.53$ (m, 5H, Ar-H), 7.68 (d, J 1/4 $15.9 \mathrm{~Hz}, 1 \mathrm{H}, \mathrm{H}-3) ;{ }^{13} \mathrm{C}$ NMR $\left(75 \mathrm{MHz}, \mathrm{CDCl}_{3}\right) \mathrm{d}$ : $14.1\left(\mathrm{C}-16^{0}\right), 22.7\left(\mathrm{C}-15^{0}\right), 25.9\left(\mathrm{C}-3^{0}\right), 28.7\left(\mathrm{C}-2^{0}\right), 29.2(\mathrm{C}-$ $\left.4^{0}\right), 29.3\left(\mathrm{C}-13^{0}\right), 29.7\left(\mathrm{C}-5^{0}-12^{0}\right), 31.9\left(\mathrm{C}-14^{0}\right), 64.7\left(\mathrm{C}-1^{0}\right), 118.3(\mathrm{C}-2)$ $128.0(\mathrm{C}-5,9), 128.8(\mathrm{C}-6,8), 130.2$ (C-4), 134.5 (C-7), 144.5 (C-3), 167.1 (C-1); HRESIMS: $m / z$ 395.2924 (M p Na) ${ }^{\mathrm{p}}$; Calcd for $\mathrm{C}_{25} \mathrm{H}_{40} \mathrm{O}_{2} \mathrm{Na}^{\text {p: }}$ : 395.2921 .

3.3.11. 3.3.Trans-octadecyl-3-(3,4-dihydroxyphenyl) propenoate (18) Colorless solid; m.p.108 C (methanol) (lit [24].110e112 C); UV (MeOH): 328, $219 \mathrm{~nm}$; IR (KBr, $\mathrm{cm}^{1}$ ): $3481(\mathrm{OH}), 3311(\mathrm{OH}), 2918$, 1684(CO),1606,1284,1180,974,815; ${ }^{1}$ HNMR(300MHz,DMSO-d 6$)$ $\mathrm{d}: 0.84\left(\mathrm{t}, J^{1 / 4} 6.6 \mathrm{~Hz}, 3 \mathrm{H}, \mathrm{H}-18^{0}\right), 1.22\left(\mathrm{bs}, 30 \mathrm{H}, \mathrm{H}-3^{0}-17^{0}\right), 1.61(\mathrm{~m}, 2 \mathrm{H}$, $\left.\mathrm{H}-2^{0}\right), 4.09\left(\mathrm{t}, \mathrm{J}^{1} / 46.45 \mathrm{~Hz}, 2 \mathrm{H}, \mathrm{H}-1^{0}\right), 6.25\left(\mathrm{~d}, J^{1 / 1} / 415.9 \mathrm{~Hz}, 1 \mathrm{H}, \mathrm{H}-2\right), 6.75$ (d, J $1 / 48.1 \mathrm{~Hz}, 1 \mathrm{H}, \mathrm{H}-8), 7.0$ (dd, J $1 / 48.1,1.8 \mathrm{~Hz}, 1 \mathrm{H}, \mathrm{H}-9), 7.04$ (d, J1/4 1.8Hz,1H,H-5), 7.45(d, J1/4 15.9Hz,1H,H-3),9.17(s,1H,OH), $9.61(\mathrm{~s}, 1 \mathrm{H}, \mathrm{OH}) ;{ }^{13} \mathrm{C}$ NMR $(75 \mathrm{MHz}$, DMSO-d 6$) \mathrm{d}: 13.4\left(\mathrm{C}-18^{0}\right), 21.6$ $\left(\mathrm{C}-17^{0}\right), 24.9\left(\mathrm{C}-3^{0}\right), 27.8\left(\mathrm{C}-2^{0}\right), 28.2\left(\mathrm{C}-4^{0}, 15^{0}\right), 28.5\left(\mathrm{C}-5^{0}-14^{0}\right), 30.8$ $\left(\mathrm{C}-16^{0}\right), 63.2\left(\mathrm{C}-1^{0}\right), 113.5(\mathrm{C}-2), 114.2(\mathrm{C}-8), 115.2(\mathrm{C}-5), 120.8(\mathrm{C}-9)$, 125.0 (C-4), 144.5 (C-3), 145.1 (C-6), 147.9 (C-7), 166.1 (C-1); HRE- SIMS: $m / z$ $455.3135(\mathrm{M} \mathrm{p} \mathrm{Na})^{\text {p }}$; Calcd for $\mathrm{C}_{27} \mathrm{H}_{44} \mathrm{O}_{4} \mathrm{Na}^{\mathrm{p}}: 455.3132$.

\subsubsection{Trans-octadecyl-3-(4-hydroxy-3-methoxyphenyl) propenoate (19)} [28].

Colorless solid; m.p.104 C (methanol) (lit [28].110 C); identical with ref

\subsubsection{Trans-octadecyl-3-(3,5-dimethoxy-4-hydroxyphenyl)} propenoate (20)

Colorless solid; m.p. 72 C (methanol) (lit [29]. 72e73 C); UV (MeOH): $327,236 \mathrm{~nm}$; IR ( $\mathrm{KBr}, \mathrm{cm}^{1}$ ): $3533(\mathrm{OH}), 2917,1708$ (CO),

1638, 1516, 1181, 836; ${ }^{1} \mathrm{H}$ NMR (300 MHz, $\left.\mathrm{CDCl}_{3}\right) \mathrm{d}: 0.87\left(\mathrm{t}, J^{1 / 4} 6.6\right.$ $\left.\mathrm{Hz}, 3 \mathrm{H}, \mathrm{H}-18^{0}\right), 1.25\left(\mathrm{bs}, 30 \mathrm{H}, \mathrm{H}-3^{0}-17^{0}\right), 1.70\left(\mathrm{~m}, 2 \mathrm{H}, \mathrm{H}-2^{0}\right)$, $3.92\left(\mathrm{~s}, 6 \mathrm{H}, 2 \mathrm{OCH}_{3}\right), 4.19\left(\mathrm{t}, J^{1 / 4} 6.6 \mathrm{~Hz}, 2 \mathrm{H}, \mathrm{H}-1^{0}\right), 5.78(\mathrm{~s}, 1 \mathrm{H}, \mathrm{OH})$, $6.30\left(\mathrm{~d}, J^{1} / 415.9 \mathrm{~Hz}, 1 \mathrm{H}, \mathrm{H}-2\right), 6.77(\mathrm{~s}, 2 \mathrm{H}, \mathrm{H}-5,9), 7.59\left(\mathrm{~d}, J^{1} / 415.9 \mathrm{~Hz}\right.$, $1 \mathrm{H}, \mathrm{H}-3) ;{ }^{13} \mathrm{CNMR}\left(75 \mathrm{MHz}^{\mathrm{C}} \mathrm{CDCl}_{3}\right) \mathrm{d}: 14.1\left(\mathrm{C}-18^{0}\right), 22.7\left(\mathrm{C}-17^{0}\right), 25.9$ $\left(\mathrm{C}-3^{0}\right), 28.8\left(\mathrm{C}-2^{0}\right), 29.2\left(\mathrm{C}-4^{0}\right), 29.3\left(\mathrm{C}-15^{0}\right), 29.7\left(\mathrm{C}-5^{0}-14^{0}\right), 31.9$ $\left(\mathrm{C}-16^{0}\right), 56.3\left(2 \mathrm{OCH}_{3}\right), 64.6\left(\mathrm{C}-1^{0}\right), 105.1(\mathrm{C}-5,9), 116.1(\mathrm{C}-2), 126.0$ (C-4), 137.1 (C-7), 144.8 (C-3), 147.2(C-6, 8), 167.2 (C-1); HRESIMS:

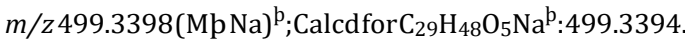

\subsubsection{Trans-octadecyl-3-phenylpropenoate (21)}

Colorless solid; m.p. 45 C (petroleum ether) (lit [29]. 45e46 C); UV $(\mathrm{MeOH}): 276,218 \mathrm{~nm} ; \mathrm{IR}\left(\mathrm{KBr}, \mathrm{cm}^{1}\right): 2920,1713(\mathrm{CO}), 1639,1311,1175,766 ;{ }^{1} \mathrm{H}$ $\mathrm{NMR}\left(300 \mathrm{MHz}, \mathrm{CDCl}_{3}\right) \mathrm{d}: 0.88\left(\mathrm{t}, \mathrm{J}^{1 / 4} 6.6 \mathrm{~Hz}, 3 \mathrm{H}, \mathrm{H}-18^{0}\right)$, $1.26\left(\mathrm{bs}, 30 \mathrm{H}, \mathrm{H}-3^{0}-17^{0}\right), 1.69\left(\mathrm{~m}, 2 \mathrm{H}, \mathrm{H}-2^{0}\right), 4.20\left(\mathrm{t}, J^{1 / 4} 6.6 \mathrm{~Hz}, 2 \mathrm{H}, \mathrm{H}-\right.$ $\left.1^{0}\right), 6.44(\mathrm{~d}, J 1 / 415.9 \mathrm{~Hz}, 1 \mathrm{H}, \mathrm{H}-2), 7.37 \mathrm{e} 7.55(\mathrm{~m}, 5 \mathrm{H}, \mathrm{Ar}-\mathrm{H}), 7.68(\mathrm{~d}, J 1 / 415.9 \mathrm{~Hz}$, $1 \mathrm{H}, \mathrm{H}-3) ;{ }^{13} \mathrm{C}$ NMR $\left(75 \mathrm{MHz}, \mathrm{CDCl}_{3}\right)$ d: $14.1\left(\mathrm{C}-18^{0}\right), 22.7$ (C-17 $\left.{ }^{0}\right), 25.9\left(\mathrm{C}-3^{0}\right)$, $28.7\left(\mathrm{C}-2^{0}\right), 29.3\left(\mathrm{C}-4^{0}\right), 29.6\left(\mathrm{C}-15^{0}\right), 29.7\left(\mathrm{C}-5^{0}-\right.$

$\left.14^{0}\right), 31.9\left(\mathrm{C}-16^{0}\right), 64.7\left(\mathrm{C}-1^{0}\right), 118.3(\mathrm{C}-2), 128.0(\mathrm{C}-5,9), 128.8(\mathrm{C}-6,8)$, 

130.2 (C-4), 134.5 (C-7), 144.5 (C-3), 167.1 (C-1); HRESIMS: $m / z$ $423.3235(\mathrm{Mp} \mathrm{Na})^{\text {p }}$; Calcd for $\mathrm{C}_{27} \mathrm{H}_{44} \mathrm{O}_{2} \mathrm{Na}^{\mathrm{p}}$ : 423.3233 .

\subsection{Determination of antioxidant activity}

(i) The DPPH assay. Total antioxidant capacity assay was per- formed using DPPH as radical source. Radical-scavenging activity was spectrophotometrically evaluated in a Powerwave XS Micro- plate Reader (Bio-Tek Instruments, Inc) by monitoring the disap- pearance of 1,1-diphenyl-2-picrylhydrazyl (DPPH) at $515 \mathrm{~nm}$, as previously reported [14]. Increasing concentrations of the different hydroxy cinnamates, were prepared in duplicate, in ethanol. Each solution $(20 \mathrm{~mL})$ was added to $180 \mathrm{~mL}$ of DPPH radical solution $(0.1 \mathrm{mM})$ in a 96 -well microplate and absorbances were recorded every minute for a $45 \mathrm{~min}$ period. The absorbance of a blank control (20 mL ethanol plus $180 \mathrm{~mL}$ of radical) was set as $100 \%$ of radical( $0 \%$ bleaching).

Radical-scavenging activity, expressed as the percentage of inhibition, was calculated according to the formula: \% Inhibition $1 / 4$ [(Acontrol Asample)/Acontrol] 100,whereAcontrol is the absorbance of the DPPH solution without the sample (blank control) and Asample is the absorbance of the tested hydrox- ycinnamate. IC50 values were calculated as the minimum concen- tration of each sample required to inhibit $50 \%$ of the DPPH radical.

(ii) The ABTS ${ }^{\mathrm{b}}$ assay.Theradical-scavengingactivityoftested compounds was measured by the Trolox equivalent antioxidant capacity (TEAC) assay, as previouslydescribed $[15,16]$.Thismethod is based on the ability of hydrogen-donating antioxidants to decolorize the preformed radical monocation of 2,2 $2^{0}$-azinobis-(3- ethylbenzothiazoline-6-sulfonic acid) (ABTS ${ }^{\mathrm{b}}$ ), generated by oxidation of ABTS with potassium persulfate. The ABTS ${ }^{b}$ solution was prepared by reaction of $10 \mathrm{~mL}$ of a 7 $\mathrm{mM}$ aqueous ABTS solu- tion and $163 \mathrm{~mL}$ of a $150 \mathrm{mM}$ (2.45 mM final concentration) potassiumpersulfatesolution. After storage in thedark for $16 \mathrm{~h}$, the radical cation solution was diluted in ethanol until the initial absorbancevalue of 0.70 .04 at $734 \mathrm{~nm}$ was reached. Solutions of 0.5 , 1.0 and $1.5 \mathrm{mM}$ for each cinnamic acid or ester were prepared (to achieve a $20 \mathrm{e} 80 \%$ decrease in the initial absorbance of the reaction solution).Analiquot of the compound solution ( $20 \mathrm{~mL}$ ) was added to $180 \mathrm{~mL}$ of the radical solution in a 96-well microplate. The decrease in absorbance was recorded at 0 and after $6 \mathrm{~min}$. Anti- oxidant concentration vs. \% absorbance reduction data were graphically plotted. The concentration of antioxidant giving the same percentage reduction of absorbance at 734 $\mathrm{nm}$ as the $1 \mathrm{mM}$ Trolox solution was calculated from the three-point graphs.

\subsubsection{Data analysis}

Each experiment was performed in triplicate. Statistical comparisons of both assays were done by one-way ANOVA fol- lowed by the multiple Duncan test $(P<0.05)$.

\subsection{Calculation of drug-likeness properties}

The parameters for drug-likeness were evaluated according to the Lipinski's 'rule-of-five', using the Molinspiration WebME Editor 1.16. [http://www.molinspiration.com].

\section{Conclusion}

Long chain alkyl hydroxy cinnamates (8e21) were synthesized from the corresponding half esters of malonic acid (5e7) and benzaldehyde derivatives by Knoevenagel condensation in good yields $(85 \%$ to quantitative). The antioxidant activity of eight hydroxycinnamyl esters was studied using DPPH and ABTS assays. The radical-scavenging ability data obtained for hydroxycinnamic esters against DPPH and ABTS radicals were in good agreement with the expected activities of this type of phenolic systems as it has been demonstrated earlier [7]: it is higher when a catechol group is present (caffeic series), and lower when the meta-hydroxyl function is substituted by a methoxyl group (ferulic series), having the sinapic series with intermediate values. The esterification does not significantly modify the antioxidantactivity of precursors, afact that is in accordance with the main objective of the work. These novel agents have the advantage of being synthesized from natural products, while providing a value-added use for oil systems. Accordingly, they could be formulated into standard UVabsorbing daily-wear cosmetic, hair and skin care and sunscreen formula- tions. Due to its antioxidant properties, lipophilicity and capacity of absorbing UVB radiation the dihydroxycinnamic derivatives could also be applied for topical formulations to treat erythema and/or other skin diseases.

\section{Acknowledgements}

We wish to thank L. D'Souza, Scientist, National Institute of Oceanography, Goa, India for recording NMR and I. Shivkumar, National Chemical Laboratory, Pune, India for HRESIMS data on our samples.

\section{References}

[1] M.C. Figueroa-Espinoza, P. Villeneuve, J. Agric, Food Chem. 53 (2005) 2779e2787. [2] H.J. Jung, S.S. Kang, S.K. Hyun, J.S. Choi, Arch. Pharm. Res. 28 (2005) 534e540. [3] Y. Takaya, Y. Kondo, T. Furukawa, M. Niwa, J. Agric, Food Chem. 51 (2003) $8061 \mathrm{e} 8066$.

[4] H.R. El-Seedi, T. Ringbom, K. Torssell, L. Bohlin, Chem. Pharm. Bull. 51 (2003) 1439e1440.

[5] M. Shiyaku, I. Koiso, M. Matsugami, T. Suzuki, Jpn. Kokai Tokkyo Koho (1989) JP 01013017 A.

[6] H. Taniguchi, E. Nomura, T. Tsuno, S. Minami, EP 0681825 A2, 1995.

[7] A. Gaspar, E.M. Garrido, M. Esteves, E. Quezada, N. Milhazes, J. Garrido, F. Borges, Eur. J. Med. Chem. 44 (2009) 2092e2099.

[8] M.A. Bernards, N.G. Lewis, Phytochemistry 31 (1992) 3409e3412.

[9] S. Venkateswarlu, M.S. Ramachandra, A.V. Krishnaraju, G. Trimurtulu, G.V. Subbaraju, Indian J. Chem. 45B (2006) 252e257.

[10] K. Kawanishi, J. Yasufuku, A. Ishikawa, Y. Hashimoto, J. Agric, Food Chem. 38 (1990) $105 \mathrm{e} 108$

[11] K.E. Kolb, K.W. Field, P.F. Schatz, J. Chem. Edu. 67 (1990) 12.

[12] A.M. D'Souza, Synthesis and transformation of some natural products and their analogues, Ph. D. Thesis, Goa University, 2002, 96.

[13] Y. Ryu, A.I. Scott, Tetrahedron Lett. 44 (2003) 7499e7502.

[14] W. Brand-Williams, M.E. Cuvelier, C. Berset, Food Sci. Technol.-LEB 28 (1995) 25e30.

[15] F.M.F. Roleira, C. Siquet, E. Orrù, E.M. Garrido, J. Garrido, N. Milhazes, G. Podda, F. Paiva-Martins, S. Reis, R.A. Carvalho, E.J. Tavares da Silva, F. Borges, Bioorg. Med. Chem. 18 (2010) 5816e5825

[16] C. Siquet, F. Paiva-Martins, J.L. Lima, S. Reis, F. Borges, Free Radic. Res. 40 (2006) $433 \mathrm{e} 442$.

[17] B. Reis, M. Martins, B. Barreto, N. Milhazes, E.M. Garrido, P. Silva, J. Garrido, F. Borges, J. Agric, Food Chem. 58 (2010) 6986e6993.

[18] M. Esteves, C. Siquet, A. Gaspar, V. Rio, J.B. Sousa, S. Reis, M.P. Marques, F. Borges, Arch. Pharm. (Weinheim) 341 (2008) 164e173.

[19] B.-M. Lue, N.S. Nielsen, C. Jacobsen, L. Hellgren, Z. Guo, X. Xu, Food Chem. 123 (2010) $221 \mathrm{e} 230$

[20] G. Vistoli, A. Pedretti, B. Testa, Drug Discov. Today 13 (7e8) (2008) 285e294. [21] C.A.

Lipinski, F. Lombardo, B.W. Dominy, P.J. Feeney, Adv. Drug Deliv. Rev. 23 (1997) $3 \mathrm{e} 25$

[22] P. Ertl, B. Rohde, P. Selzer, J. Med. Chem. 43 (2000) 3714e3717.

[23] D. Davidson, S.A. Bernhardt, J. Am. Chem. Soc. 70 (1948) 3426e3428.

[24] K. Uwai, Y. Osanai, T. Imaizumi, S. Kanno, M. Takeshita, M. Ishikawa, Bioorg. Med. Chem. 16 (2008) 7795e7803.

[25] A.E. Nkengfack, T.W. Vouffo, J.C. Vardamides, J. Kouam, Z.T. Fomum, M. Meyer, O. Sterner, Phytochemistry 46 (1997) 573e578.

[26] K. Vosmann, P. Weitkamp, N. Weber, J. Agric, Food Chem. 54 (2006) 2969e2976.

[27] V. Ramesh, R.G. Weiss, J. Org. Chem. 51 (1986) 2535e2539.

[28] B.T. Ngadjui, E. Dongo, E.N. Happi, M.T. Bezabih, B.M. Abegaz, Phytochemistry 48 (1998) $733 \mathrm{e} 737$.

[29] K. Nishimura, Y. Takenaka, M. Kishi, T. Tanahashi, H. Yoshida, C. Okuda Y. Mizushina, Chem. Pharm. Bull. 57 (2009) 476e480. 